\title{
Radiotherapy and Complementary Medicine in Palliative Care
}

\author{
Angélica Lopes ${ }^{1}$, Diogo Amorim² , Inês Espiga Macedo ${ }^{2}$ and José Carlos Martins ${ }^{3 *}$ \\ ${ }^{1}$ Radiation Terapist, Master in Palliative Care, Portugal \\ ${ }^{2}$ Instituição: Instituto de Medicina Integrativa, Coimbra, Portugal \\ ${ }^{3}$ Coordinator Professor at the Nursing School of Coimbra, Portugal
}

Submission: April 09, 2018; Published: May 04, 2018

*Corresponding author: José Carlos Amado Martins, Coordinator Professor at the Nursing School of Coimbra, Coimbra, Master in Palliative Care, Portugal, Email: angelica.calopes@gmail.com

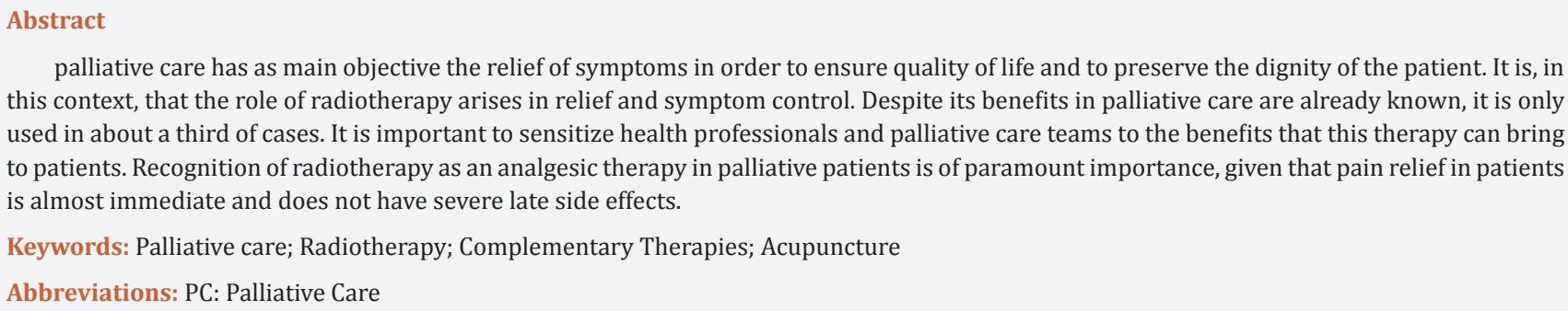

palliative care has as main objective the relief of symptoms in order to ensure quality of life and to preserve the dignity of the patient. It is, in this context, that the role of radiotherapy arises in relief and symptom control. Despite its benefits in palliative care are already known, it is only used in about a third of cases. It is important to sensitize health professionals and palliative care teams to the benefits that this therapy can bring to patients. Recognition of radiotherapy as an analgesic therapy in palliative patients is of paramount importance, given that pain relief in patients is almost immediate and does not have severe late side effects.

Keywords: Palliative care; Radiotherapy; Complementary Therapies; Acupuncture

Abbreviations: PC: Palliative Care

\section{Introduction}

The use of radiation therapy in palliative care is not a recent practice. Its use in palliative care comes together with the initial studies when applying radioactivity for medical purposes. The World Health Organization defines palliative care as an active response to the problems of prolonged, incurable and progressive illness, in an attempt to prevent the suffering it causes and to provide the maximum possible quality of life for these patients and their families. In these care there is a combination of science and humanism [1].

The evolution of the means of diagnosis and treatment has led to an increase in the quality of life as well as the average life expectancy. Yet a question had to be asked: what if healing was not possible and death inevitable? The World Health Organization forecasts that every year, 20 million people will need Palliative Care (CP). Modern palliative care, which emerged around the 1960s at St Christopher's and aims to improve the quality of life of the patient by focusing on key aspects such as symptom control, rehabilitation, continuous care, terminal care, the well-being like your family starting at the time of diagnosis and will end with the mourning phase. The need to create care capable of responding to these cases in the most extensive way possible and to do so requires a multidisciplinary team and it is in this sense that radiotherapy appears in palliative care.
Radiotherapy is a medical specialty that uses ionizing radiation in a controlled manner, with therapeutic objectives, in neoplastic lesions. The main objective of radiotherapy is to treat the target volume and to preserve tissue as much as possible adjacent ones. Radiation therapy can be classified into external radiation therapy when it uses an external radiation source or in brachytherapy when the radioactive source is placed in direct contact with the target volume. In external radiotherapy, the total dose is given in small fractions daily over a variable period of weeks depending on the fractionation chosen for the treatment [2].

Early records on the benefits of palliative radiotherapy date back to 1930 and were used primarily in patients with bone metastases.

The main objective of the use of radiotherapy in palliative care is the control of symptoms that interfere in the patient quality of life of the, monitoring and accompanying the patient's well-being through a daily evaluation, using a multidisciplinary team. Palliative radiotherapy has as main objective the control of symptoms, such as pain, hemorrhage and occlusive syndromes, being its main indications for bone metastases (when associated with a high risk of fracture) pain or compression of other organs (medullary compression), cerebral, pulmonary or hepatic 
metastases and ulcerated tumors. Radiotherapy is an effective, quick-acting palliative and less expensive treatment compared to continuous analgesic use.

The radiotherapy intervention can be divided in three groups:

i. Anti-cyclic, cyto-reductive and decompressive radiotherapy, aimed at relieving pain and / or reducing infiltrative, uncomfortable, organic or ulcerated tumor mass (eg brain metastases, bulky and painful tumors);

ii. Prophylactic radiotherapy, refers to bone consolidation, in a way to prevent pathological fractures and eventual compressive phenomena;

iii. Hemostatic therapy, it is the control of uncontrollable hemorrhages (eg. gynecological hemorrhages).

Radiotherapy plays an important role in palliative patients in relieving pain. This is one of the most distressing symptoms for patients and their families, as it interferes with simple day-to-day activities, the capacity for self-care and can trigger symptoms such as insomnia, depression, anorexia and others. Studies indicate that about $60-70 \%$ of patients undergoing palliative radiotherapy feel a gradual relief of pain after treatment. However, the prognosis of the patient should be taken into account when radiotherapy is prescribed, since the benefits of radiotherapy generally only begin after 2-4 weeks of treatment. However, it is necessary to take into account that this therapy has some side effects, namely the acute ones that occur during the treatment, being predictable and reversible. These late side effects appear months or years after radiotherapy and are unpredictable. There is therefore a need to adjust the whole treatment process to the patient's performance status.

Another aspect to take into account in a palliative patient who is doing radiotherapy are the interruptions. Since one of the principles of radiotherapy and radiological calculation is to administer the prescribed total dose in the chosen fractionation without prolonging the total treatment, time in order to ensure its efficiency. Interruptions throughout treatment may affect local control and cure rate, hence the importance of cure as short as possible. It is therefore important to personalize the treatment taking into account the needs and limitations of each patient, making it essential that the patient is informed and participates in the decisions related to the treatment parameters.

Since radiotherapy is advisable between $50 \%$ and $80 \%$ of the palliative cases and presenting a low level of toxicity to the patient, it is used in about $35 \%$ of the cases [3]. To justify the low percentage of palliative patients who resort to this treatment modality, logistic factors and factors associated with the patient itself are applied.

It is important to sensitize health professionals and palliative care teams to the benefits that this therapy can bring to patients. Recognition of radiotherapy as an analgesic therapy in palliative patients is of paramount importance, given that pain relief in patients is almost immediate and does not have severe late side effects. Another advantage that radiotherapy presents in relieving pain is that this is a relatively short treatment since the days of treatment are usually calculated based on the patient's performance status.

\section{What Is The Role Of Complementary Medicine And Alternative Therapies?}

Integrative medicine definition vary widely, for most institutions it is considered an approach that brings together conventional and complementary therapies, based on the best evidence available.

There is a growing discussion about the role of integrative medicine in Palliative care, namely because of its focus on improving function and quality of life through a biopsychosocial approach $[2,4]$. The available evidence, in the form of primary research, points out for an important role of complementary therapies and integrative medicine approach in the palliative care setting, however, its complexity as an intervention needs to be acknowledged in future research designs and when reviewing research findings [5]. In palliative care symptoms vary from patient to patient depending on their disease course, however there are some symptoms which are common to a group of diseases. The side effects of chemotherapy and radiotherapy such as nausea, vomiting, leucopenia and xerostomia, and the cancer-related symptoms such as pain, fatigue, insomnia are some of the symptoms that are common towards the end of life.

Most of the current evidence is about the use of acupuncture, acupressure and electroacupuncture in palliative care.

Acupuncture results in a stimulation of deep tissue sensory afferent nerves as the initial event leading to the activation of the central nervous system pathways that allow a neuromodulation of the sensory and autonomic system. In various animal models this neuromodulation has resulted in a range of physiological responses including regulation of blood pressure, gut motility, immune responses, glucose metabolism, glucocorticoids, and sex hormones [6].

There is evidence for the therapeutic effects of acupuncture for patients with cancer on reduction of cancer related fatigue, chemotherapy-induced vomiting and pain. There is conflicting evidence about the role of acupuncture in chemotherapy-induced nausea, leucopenia, vasomotor symptoms and improving quality of life with most of the reviews pointing for positive results compared to conventional care alone. There is insufficient evidence to support or refute the potential of acupuncture and related therapies in the management of xerostomia and lymphedema $[2,6,7]$.

The use of additional therapies as aromatherapy, Reiki, massage therapy, animal therapy, relaxation techniques, music therapy, tai chi, exercise and cognitive behavioral therapy can 
have a central role on Palliative care, being part of some of the international recommendations [2,4,8-12]. Recent studies have shown therapeutic effects of aromatherapy, Reiki, massage therapy, animal therapy, relaxation techniques, music therapy for fatigue, depression, anxiety and stress. Symptoms which lead to suffering, diminished quality of life and global survival [8,912]. Clinical trials have shown that there is a better control of dyspnea in palliative care when reflexology13 and acupuncture are used as adjuvant care [13].

By contributing to the reduction of the side effects of the conventional therapies and by increasing the general wellbeing of the patients, integrative medicine and complementary therapies may allow them to achieve a better outcome from the overall treatment namely by increasing the tolerance to chemotherapy and radiotherapy treatments $[14,15]$.

Complementary therapies have been growing all over the world, either on hospital setting or in dedicated centers of treatment out of the traditional care setting. There is a need of knowledge of the different therapies available, their methodological basis and known mean of action as well as the best evidence available for their therapeutic effects in the young health professional in order to make it possible to choose the best therapies available [16,17].

\section{Conclusion}

With this short article, we hope to have made clear the space available for radiotherapy and complementary therapies in palliative care. Both have high potential to improve the patient's quality of life, mainly through the control of symptoms.

\section{References}

1. World Health Organization (2016) Cancer: WHO definition of palliative care. WHO, Geneva, Switzerland.

2. Washington C, Leaver D, (2016) Principles and practice of radiation therapy. ( $4^{\text {th }}$ edn), St Louis, Elsevier, pp. 10-12.

3. Tiwana M, Barnes M, Kiraly A, Olson R, (2016) Utilization of palliative radiotherapy for bone metastases near end of life in a populationbased cohort. BMC Palliative Care, doi: 10.1186/s12904-015-0072-5.
4. Kogan M, Cheng S, Rao S, DeMocker S, Koroma Nelson M (2017) Integrative Medicine for Geriatric and Palliative Care. Med Clin North Am 101(5): 1005-1029.

5. Towler P, Molassiotis A, Brearley SG (2013) What is the evidence for the use of acupuncture as an intervention for symptom management in cancer supportive and palliative care: an integrative overview of reviews. Support Care Cancer 21(10): 2913-2923.

6. Zia FZ, Olaku O, Bao T, Berger A, Deng G, et al. (2017) The National Cancer Institute's Conference on Acupuncture for Symptom Management in Oncology: State of the Science, Evidence, and Research Gaps. J Natl Cancer Inst Monogr, doi: 10.1093/jncimonographs/lgx005.

7. Lian WL, Pan MQ Zhou DH, Zhang ZJ (2014) Effectiveness of acupuncture for palliative care in cancer patients: a systematic review. Chin J Integr Med 20(2): 136-147.

8. Barati F Nasiri A, Akbari N, Sharifzadeh G (2016) The Effect of Aromatherapy on Anxiety in Patients. Nephrourol Mon 8(5): e38347.

9. Caires J (2014) The use of Complementary Therapies in palliative care: benefits. Cogitare Enferm, 471-477.

10. Diniz A (2015) Terapias Complementares em Cuidados Paliativos Pediátricos. Universidade Atlantida, Projeto final de Licenciatura.

11. Graner K, Costa AL, Rolim GS (2010) Dor em oncologia: intervenções complementares e alternativas ao tratamento medicamentoso. Temasem Psicologia 18(2): 34-35.

12. Hubner J (2013) Sport against cancer-related fatigue. Dtsch med Wochenschr 38(3): 353.

13. Romeo MJ, Parton B, Russo RA, Hays LS, Conboy L (2015) Acupuncture to Treat the Symptoms of Patients in a Palliative Care Setting. Explore 11(5): 357-362.

14. Associação portuguesa de cuidados paliativos. Cuidados paliativos: o que são?

15. Cheng CS, Chen LY, Ning ZY, Zhang CY, Chen H, et al. (2017) Acupuncture for cancer-related fatigue in lung cancer patients: a randomized, double blind, placebo-controlled pilot trial. Support Care Cancer 25(12): 3807-3814.

16. Steinhorn DM, Din J, Johnson A (2017) Healing, spirituality and integrative medicine. Ann Palliat Med 6(3): 237-247.

17. Wyatt, G, Sikorskii A, Rahbar MH, Victorson D, You M (2012). HealthRelated Quality-of-Life Outcomes: a reflexology trial with patients with advanced-stage breast cancer. Oncol Nurs Forum 39(6): 568-577.

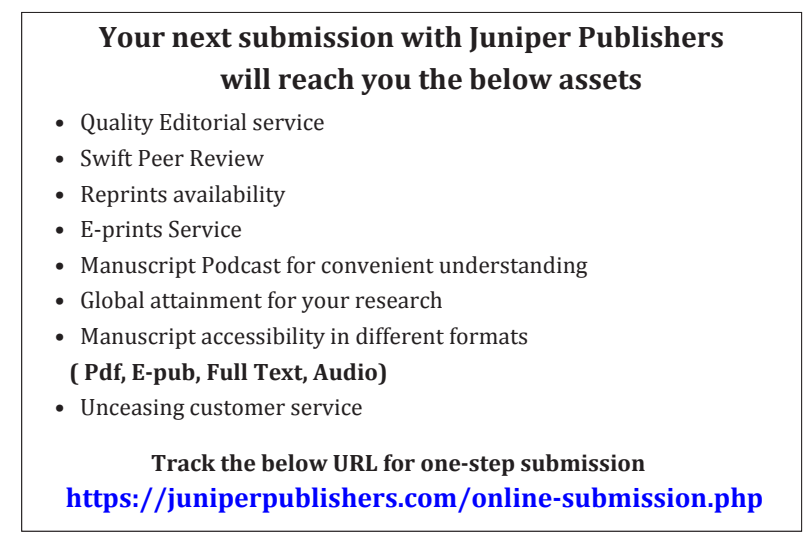

